\title{
A comparison of population estimation techniques for individually unidentifiable free-roaming dogs
}

\author{
N. V. Meunier ${ }^{1}$, A. D. Gibson ${ }^{2,3}$, J. Corfmat ${ }^{2}$, S. Mazeri ${ }^{2,3}$, I. G. Handel' ${ }^{1}$, L. Gamble ${ }^{2}$, B Mde C Bronsvoort ${ }^{3}$ and \\ R. J. Mellanby ${ }^{1 *}$ id
}

\begin{abstract}
Background: Measuring the size of free roaming dog populations quickly and accurately is critical in the implementation of numerous preventive health and population control interventions. However, few studies have investigated the relative performance of population size assessment tools when applied to dogs. The aim of this study was to compare the commonly used mark-resight methodology with distance sampling methods, which are less resource intensive, to estimate free-roaming dog abundance in Goa, India.

Twenty-six working zones were surveyed along all roads twice by the same surveyor at the same time of day, following a vaccination campaign which included marking of vaccinated dogs with a coloured paint. The Chapman estimate was then used to evaluate the mark-resight abundance. Additionally, the number of dogs and perpendicular distance from the road for all dogs sighted was recorded. This was used to estimate dog density and abundance using distance sampling methods. The detection function was fitted based on goodness-of-fit and AIC.

Results: The Chapman abundance estimate for the entire study area was 5202 dogs (95\%Cl 4733.8-5671.0), and the distance sampling method abundance estimate was 5067 dogs $(95 \% \mathrm{Cl} 4454.3-5764.2)$. For individual working zones, after taking other factors into account in a mixed effects model, the average distance sampling estimate was $35 \%$ higher (95\% Cl 20-53\%) than the Chapman estimate. There was also evidence of a difference in estimates between surveyors of $21 \%(95 \% \mathrm{Cl} 7-37 \%)$ and between days (22\% lower on day $2,95 \% \mathrm{Cl} 8-38 \%)$ for individual working zones.
\end{abstract}

Conclusion: Our study demonstrated that the distance sampling estimates were comparable overall to the Chapman method of abundance estimation of free roaming dogs across the entire study region but there was noticeable variation between the two methods when individual zones were compared. Consequently, distance sampling methods may be suitable to enumerate dogs over large areas in a more time efficient manner than the widely used mark-resight approach.

Keywords: Population estimation, Dog abundance, Free-roaming dogs, Mark resight

\section{Background}

Domestic dogs are the source of almost all human rabies cases [1], but mass vaccination of dogs has been shown to dramatically reduce canine rabies, and subsequently human cases [2]. In addition to mass vaccination, the World Health Organisation recommends animal birth control

\footnotetext{
* Correspondence: Richard.Mellanby@ed.ac.uk

${ }^{1}$ The Royal (Dick) School of Veterinary Studies (R(D)SVS) and the Roslin Institute, Hospital for Small Animals, Easter Bush Veterinary Centre, Midlothian EH25 9RG, Scotland

Full list of author information is available at the end of the article
}

programmes to reduce stray dog populations $[3,4]$. The vaccination coverage target is accepted to be $70 \%$, which was modelled as effective to control the transmission of the rabies virus [5], with many campaigns meeting this target [6]. However, the main challenge in vaccination campaigns is to meet a sufficient vaccination coverage of the dog population [7] and to measure this impact. In order to successfully implement these vaccination programmes, there is a clear need to be able to estimate the size of dog populations quickly and accurately.

(c) The Author(s). 2019 Open Access This article is distributed under the terms of the Creative Commons Attribution 4.0 International License (http://creativecommons.org/licenses/by/4.0/), which permits unrestricted use, distribution, and reproduction in any medium, provided you give appropriate credit to the original author(s) and the source, provide a link to the Creative Commons license, and indicate if changes were made. The Creative Commons Public Domain Dedication waiver (http://creativecommons.org/publicdomain/zero/1.0/) applies to the data made available in this article, unless otherwise stated. 
The World Society for the Protection of Animals, and the Rabies Blueprint produce survey guidelines for measuring the abundance of dogs and vaccination coverage after a rabies vaccination campaign $[8,9]$. These include door-to-door surveys, sampling, indicator counts, and capture-mark-recapture methods. Methods looking at absolute abundance are time and resource intensive and this can limit their use for regular population studies [10]. Indicators, such as repeated direct counts along prescribed routes [11], are more feasible for longitudinal studies, but give no indication of total abundance. Enumerating dogs is important for planning operational activities at the start of a vaccination or sterilisation campaign, and serve as a benchmark for population interventions. Additionally, abundance estimates can be used to calculate vaccination coverage as a measure of effectiveness of the interventions.

Free-roaming dogs, i.e. dogs that are not restricted in their movements, can be difficult to enumerate if they are unowned and traditional door-to-door surveys may not accurately account for these dogs. Household surveys are therefore effective in certain countries such as Tanzania, Malawi, and Chad with lower unowned populations but may be less suitable for areas with a significant unowned, free-roaming population such as in India $[1,6,12]$. Free-roaming dogs have been described as difficult to catch and vaccinate, serving as a reservoir for rabies [7]. Understanding, this free-roaming population is therefore important for the control of infectious diseases [13].

Good enumeration techniques are available in ecological studies and ideally involve identification of individual animals [14]. Arguably, the most commonly used approaches are variations on the capture-mark-recapture methodology, for which multiple analysis techniques have been developed. This involves initially capturing and marking animals, which can be either physically restrained and marked with, for example, dyes, collars or ear-notching, or photographic capture, as was pioneered in stray dogs by Beck (1973) [15]. Animals are then released and recaptured or resighted on a second occasion, with identification of the marks. Individual identification can be resource intensive e.g. photographic comparisons $[16,17]$ and not well suited to the operational practicalities of mass vaccination campaigns, especially with a large unowned proportion. Additionally, physical marking of stray animals may not be possible without the large resources required for capture.

Distance sampling techniques do not require capture or marking of animals. These rely on measuring the perpendicular distance of the animal from randomly placed transects through the study area. These methods have been infrequently used for dog population estimation but have been reported in the Philippines [18]. Our study wished to explore a simple distance sampling technique for estimating the abundance of free-roaming dogs when individual animals could not be identified.

The aim of this study was to compare the frequently used mark-resight methodology against a less time and resource intensive distance sampling technique to enumerate the size of free roaming dog populations in regions of Goa, India. Secondly, we wished to investigate if surveys using a subset of roads for these estimations would give similar results.

\section{Materials and methods Study site}

The study took place in the Tiswadi taluka of the state of Goa in south west India, following a vaccination project by the Mission Rabies charity during March and April 2018 (Fig. 1). Each panchayat (ward) of the region was further subdivided, based on housing density, into smaller working zones which could be covered by a vaccination or survey team within one to three days. Goa administrative boundary data were obtained from GADM (https://gadm.org/data.html). All working zones in which vaccination activities occurred during the study period were considered for inclusion in the study. These were selected based on the logistics of the campaign and were considered typical for the region. The study area covered a range of settlement types from rural villages to urban areas. The average population and number of households per panchayat included in the study were not statistically different to all panchayats in Goa. Dogs were classified as free-roaming if they were seen on the streets or were in yards with access to the street. Dogs within houses, behind closed gates, or tied within a property, were considered confined and were outside the scope of this study.

\section{Marking methods}

Vaccination teams of 5-8 individuals, were allocated working zones and instructed to vaccinate all dogs within the demarcated area covering all accessible roads. Each rabies vaccination was recorded on a designated smartphone app (WVS Data collection App), capturing details on sex, age, confinement status, and GPS location $[19,20]$. Dogs were marked with a coloured paint before release. Free-roaming dogs were captured by hand or by net, depending on their temperament. After completion of a working zone by one team, the area was revisited, either on the same day or the following day, by another vaccination team in an attempt to vaccinate any missed dogs.

\section{Survey techniques}

Sight surveys were conducted in each working zone within $48 \mathrm{~h}$ after vaccination. Surveys were conducted by a single surveyor travelling by moped, instructed to 


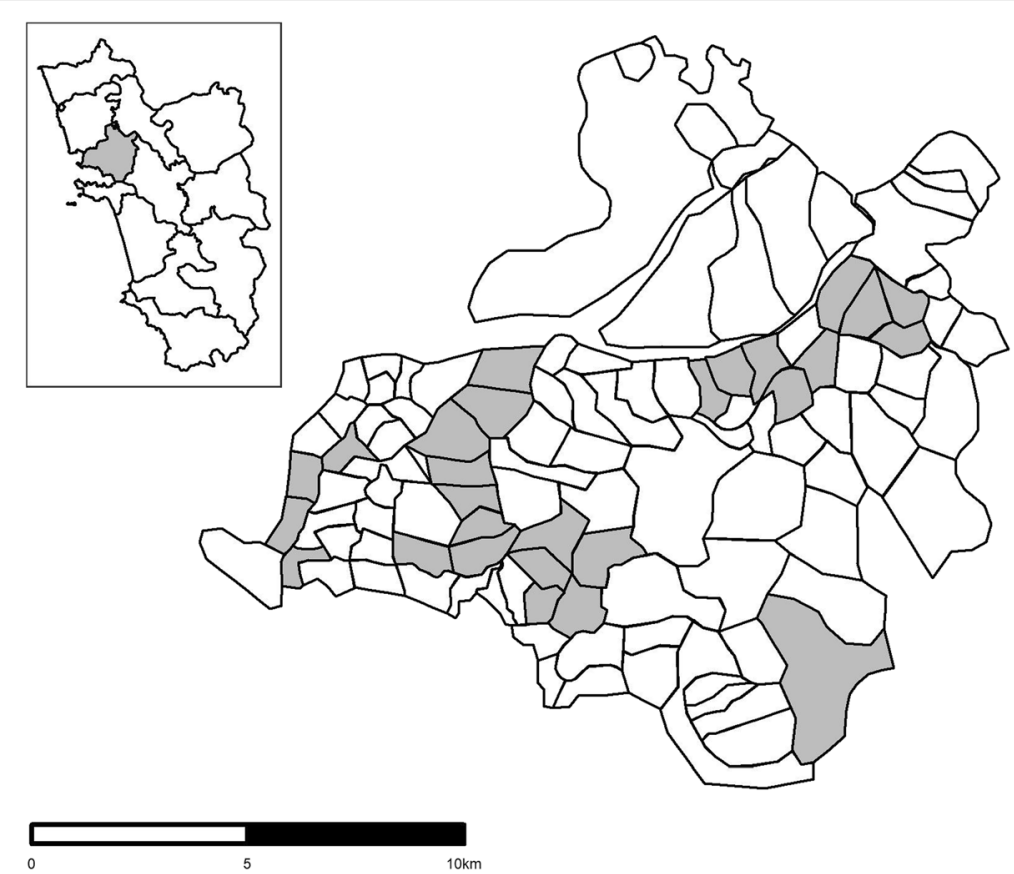

Fig. 1 (Inset) Map of Goa, showing Tiswadi taluka in grey, (Main) Tiswadi taluka showing working zones highlighted in grey $(n=26)$

travel down every street of the designated working zone to sight dogs. Surveys were completed either in the morning or afternoon, and were repeated by the same surveyor, at the same time on the following day, following the same routes. For each sighted dog, the surveyor entered the sex, age, and mark status (paint mark present or absent) of a dog into the smart phone app, which also recorded the GPS location. Only freeroaming dogs were recorded. Additionally, the surveyor estimated the perpendicular distance of the dogs from the centre of the road, as well as the road width.
Surveyors were trained before the study to estimate distances, with weekly refreshers during the study period.

At the same time as the main survey, another surveyor sighted dogs along a pre-specified subset of roads in the same area, at the same time of day. This was done for the purpose of evaluating the agreement in the estimates between the standard estimation techniques along all roads, and using a subset of roads which would be less resource and time intensive. The subset of roads was available on a map to the surveyors via the smart phone app (Fig. 2). These were selected before the study by

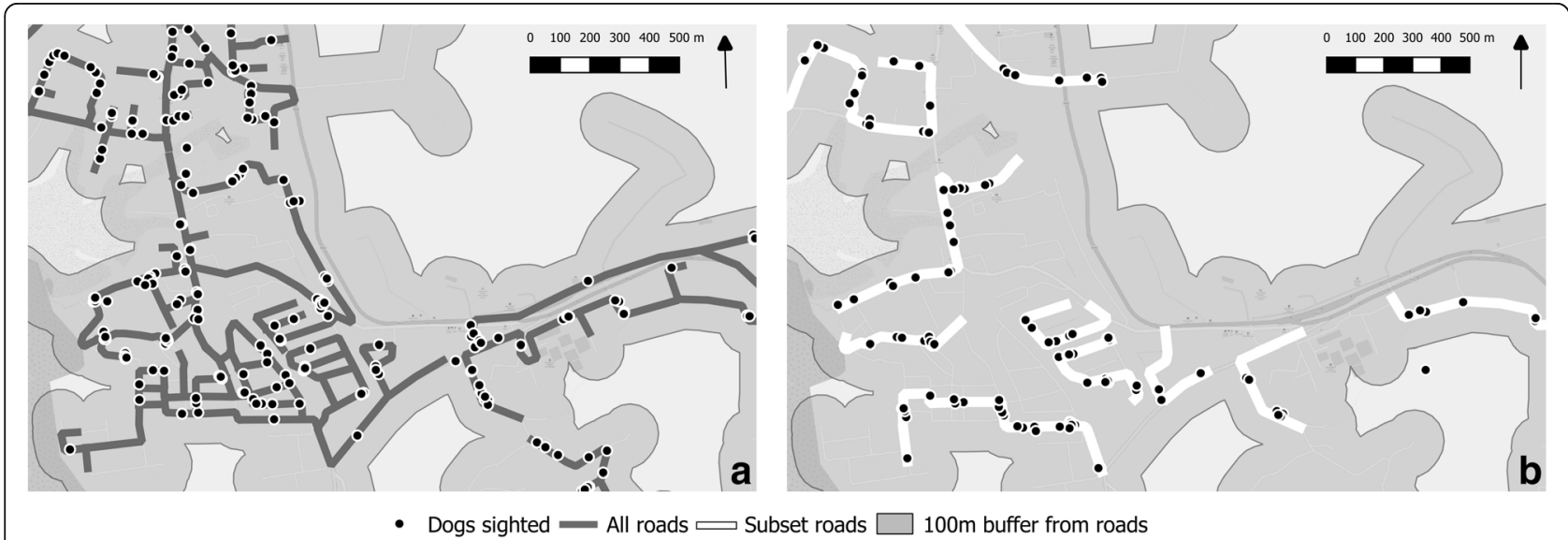

Fig. 2 Close-up map of a typical study area, showing routes covered during the survey a) all roads, b) subset roads, dogs sighted (black points), and the study area buffer within $100 \mathrm{~m}$ of roads (grey shading). Dogs were not expected to be seen outside of the buffer areas. Adapted from OpenStreetMap 
attempting to select roads along a systematic zig-zag pattern per area based on the OpenStreetMap [21] road vector map. This included main roads as well as smaller residential roads and paths. Surveyors followed a rota to switch between surveying all roads or the subset, so that both techniques would be covered by all surveyors.

The 26 working zones covered an area of $20.94 \mathrm{~km}^{2}$ within $100 \mathrm{~m}$ of the roads network excluding forested or farming land. The road network covered $100.91 \mathrm{~km}$ and the subset of roads covered a network of $47.20 \mathrm{~km}$ of roads. Each work zone was surveyed 4 times (all roads and a subset of roads, repeated the following day) with a total of 104 surveys. Two trained surveyors covered the bulk of the surveys with an additional trained surveyor conducting 4 surveys due to staff illness. A further 11 work zones were surveyed but not included in the study due to incomplete surveys or repeated surveys not conducted because of staff illness or redeployment, or because data were lost owing to technical difficulties on the smartphones.

\section{Analysis \\ Population estimation}

The sight surveys were analysed using the mark-resight Chapman estimate, to estimate dog abundance, taking the proportion of marked and unmarked dogs into account. The estimates were calculated for each survey per work area and totalled across all areas. Averages of the two surveys per area were also calculated.

The Chapman estimate is given by [22]:

$$
N=\left[\frac{\left(n_{1}+1\right)\left(n_{2}+1\right)}{(m+1)}\right]-1
$$

Where $N$ is the population size being estimated, $n_{1}$ is the number of animals initially marked, $n_{2}$ is the number of animals sighted during the follow-up survey, and $m$ is the number of marked animals sighted in the follow-up survey.

The perpendicular distances of dogs to the roads were analysed in $\mathrm{R}$ with the package Distance [23], to estimate, firstly, dog density per area and secondly, dog population abundance. The transect line was considered to be $1 \mathrm{~m}$ inside the road edge and the perpendicular distance measured to this line. Any dogs in the centre of the road were assigned as being on this transect line. The centre of the road was usually avoided by dogs due to car traffic, and they tended to rest along the road edge. The detection function models for the distance sampling method were selected by AIC, after plausible selection on Chi-square goodness-of-fit tests and visualising the function shape, with varying adjustment terms (cosine, simple polynomial, hermite polynomial). Distances measured were grouped in $5 \mathrm{~m}$ categories centred on multiples of five to avoid increased frequencies of easily reported numbers. The truncated distance was $37.5 \mathrm{~m}$. The final detection function model key functions were half-normal with cosine adjustments for the data from all roads, and hazard-rate with independent variables for the subset road data. The final models are listed in the additional materials (Additional file 1: Table S1).

The area used to estimate the abundance was restricted to within $100 \mathrm{~m}$ of a road. This was calculated in QGIS [24] by adding a $100 \mathrm{~m}$ buffer around all known roads from a OpenStreetMap vector map of the area [21], to calculate reasonably likely areas (in metres squared) where dogs would be found. This was confirmed on satellite image overlay to exclude forested areas or fields but include all built up areas.

\section{Comparative analysis}

A comparative analysis was firstly conducted of the numerical estimates for the dog population abundance between the Chapman and distance sampling methods. Secondly, we compared the estimates between using all roads and the subset of roads.

Lin's Concordance Coefficient was calculated for the comparisons and Bland-Altman plots (Additional file 2: Figure S1) allowing visualisation of the patterns of agreement between the methods [25, 26].

A mixed-effects regression model was used to analyse factors associated with the estimated abundance. The calculated abundance estimate was log transformed as the outcome measure for the final mixed-effects model. The final fixed effects were the type of estimate (Chapman or distance-method), surveyor, first or second survey, all road or subset survey, and time of day. The work zone was considered a random effect. No interactions were selected for the final model. The final mixed model was selected based on AIC and the residuals were visually assessed.

\section{Results}

\section{Comparison at study level}

A summary of the abundance estimates for the 26 work zones evaluated are given in Table 1, divided into surveys for all roads and the subset roads, as well as the first and second survey. For the surveys that covered all roads, the standard technique, the average proportion of marked dogs was 0.45 (SD 0.22) with an average of 23 dogs (SD 10) seen per area. The Chapman abundance estimate for the entire study area considering data from all roads was 5202 dogs (95\%CI 4733.8-5671.0), and the distance sampling method estimate was 5067 dogs (95\%CI 4454.3-5764.2). The density estimate from the Distance software was 242 dogs per $\mathrm{km}^{2}$ (95\%CI $213-$ 275) for all roads as transects, and an average of 6 dogs (SD 1.9) were seen per $\mathrm{km}$ of road covered. 
Table 1 Summary of surveys giving mean mark proportion per working zone, mean number of dogs sighted per working zone and total across areas, Chapman and distance sampling method estimates with 95\% confidence intervals. In bold: Means of repeated surveys, abundance estimates calculated with data from both surveys

\begin{tabular}{|c|c|c|c|c|c|c|c|c|c|}
\hline & \multirow{2}{*}{\multicolumn{2}{|c|}{$\frac{\text { Mark proportion }}{\text { Per working zone }}$}} & \multicolumn{3}{|c|}{ Number of dogs sighted } & \multirow{2}{*}{\multicolumn{2}{|c|}{$\frac{\text { Chapman abundance }}{\text { Total study area }}$}} & \multirow{2}{*}{\multicolumn{2}{|c|}{$\begin{array}{l}\text { Distance-method abundance } \\
\text { Total study area }\end{array}$}} \\
\hline & & & \multirow{2}{*}{$\begin{array}{l}\text { Total } \\
\text { area }\end{array}$} & \multicolumn{2}{|c|}{ Per working zone } & & & & \\
\hline & Mean & $(\mathrm{SD})$ & & Mean & (SD) & Estimate & $(95 \% \mathrm{Cl})$ & Estimate & $(95 \% \mathrm{Cl})$ \\
\hline \multicolumn{10}{|l|}{ All roads } \\
\hline Both surveys & 0.45 & $(0.22)$ & & 22.63 & $(9.70)$ & 5202.36 & $(4733.75-5670.97)$ & 5067.06 & $(4454.27-5764.17)$ \\
\hline Survey 1 & 0.41 & $(0.25)$ & 559 & 21.42 & $(9.27)$ & 6438.73 & $(6044.40-6833.07)$ & 5681.90 & $(4945.60-6527.81)$ \\
\hline Survey 2 & 0.48 & $(0.18)$ & 622 & 23.84 & $(10.15)$ & 3965.99 & $(3683.61-4248.36)$ & 4948.98 & (4296.05-5701.14) \\
\hline \multicolumn{10}{|l|}{ Subset roads } \\
\hline Both surveys & 0.41 & $(0.23)$ & & 13.65 & $(5.83)$ & 5190.76 & $(4491.27-5890.26)$ & 5810.61 & (5141.12-6567.28) \\
\hline Survey 1 & 0.40 & $(0.24)$ & 359 & 13.77 & $(5.02)$ & 5743.77 & (5239.96-6247.59) & 7019.16 & $(5529.45-8910.22)$ \\
\hline Survey 2 & 0.42 & $(0.22)$ & 358 & 13.53 & $(6.64)$ & 4637.75 & $(4157.64-5117.87)$ & 5295.03 & $(4385.32-6393.45)$ \\
\hline
\end{tabular}

For the distance sampling method estimates, the perpendicular distance of a dog from the road edge (transect) was measured. These distances are summarised in Fig. 3, with the majority of distances being less than $20 \mathrm{~m}$ from a road edge. Increased frequency of values centred on multiples of 5 can be seen due to the estimates made by the surveyors and the data were therefore categorised for selecting the detection function. Anecdotal evidence suggested that housing, walls, and dense bush often prevented detecting dogs beyond this $20 \mathrm{~m}$ distance.

\section{Comparison at work zone level}

The abundance estimates are visualised per work zone in Fig. 4, plotted against the number of dogs vaccinated

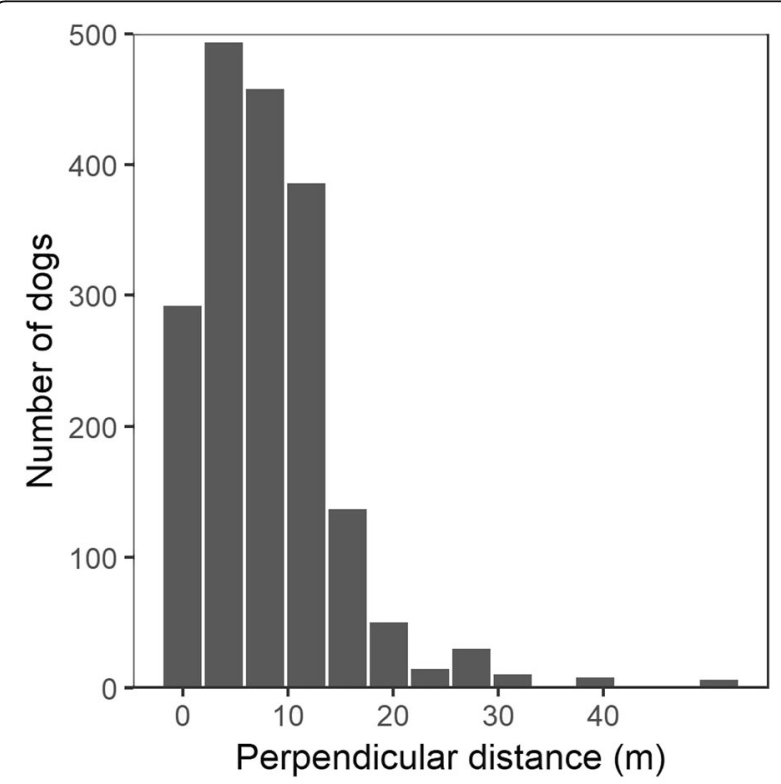

Fig. 3 Histogram of the perpendicular distance a dog was sighted away from the road per area for the first all road survey. The order from left to right is in decreasing proportion of marked dogs sighted and as the marked proportion decreases, the Chapman confidence intervals become larger.

On average, the Chapman abundance estimate was 20.76 lower $(95 \% \mathrm{CI}-58.53,17.01)$ than the distance sampling method estimate, which can be seen visually in the skewness of the scatterplot between these measures (Fig. 5a). Lin's concordance correlation coefficient (CCC) was 0.17 , which indicates a poor agreement between these measures at the work zone level without adjustment.

From the mixed-effects model, the expected dog abundance estimate per working zone for the first morning, all-road survey using the Chapman method was 158 dogs (Table 2). The distance sampling method estimate was $35 \%$ higher $(95 \% \mathrm{CI} 20-53 \%)$ than the Chapman estimate taking the surveyors, survey order, survey type and time of day into account. With the sampling method used as a co-factor in the model, there was also evidence of a difference between surveyors of $21 \%$ (95\%CI $7-$ $37 \%$ ) and between days ( $22 \%$ lower on day 2 , 95\% CI 8 $38 \%)$. There was no evidence of a difference between all road and subset road surveys, or surveys conducted in the morning or afternoon.

\section{Comparison of full survey versus subset of roads}

In the surveys that only covered a subset of roads, the mean marked proportion of dogs was 0.41 (SD 0.23), with an average of 14 dogs (SD 6) per survey. The abundance estimate for the Chapman technique for the study area considering only the data from the surveys covering the subset of roads was 5191 dogs $(95 \%$ CI $4491.3-$ 5890.3), with the distance sampling method estimating 5811 dogs (95\%CI 5141.1-6567.3). The density estimate from the Distance software was 277 dogs per $\mathrm{km}^{2}$ 


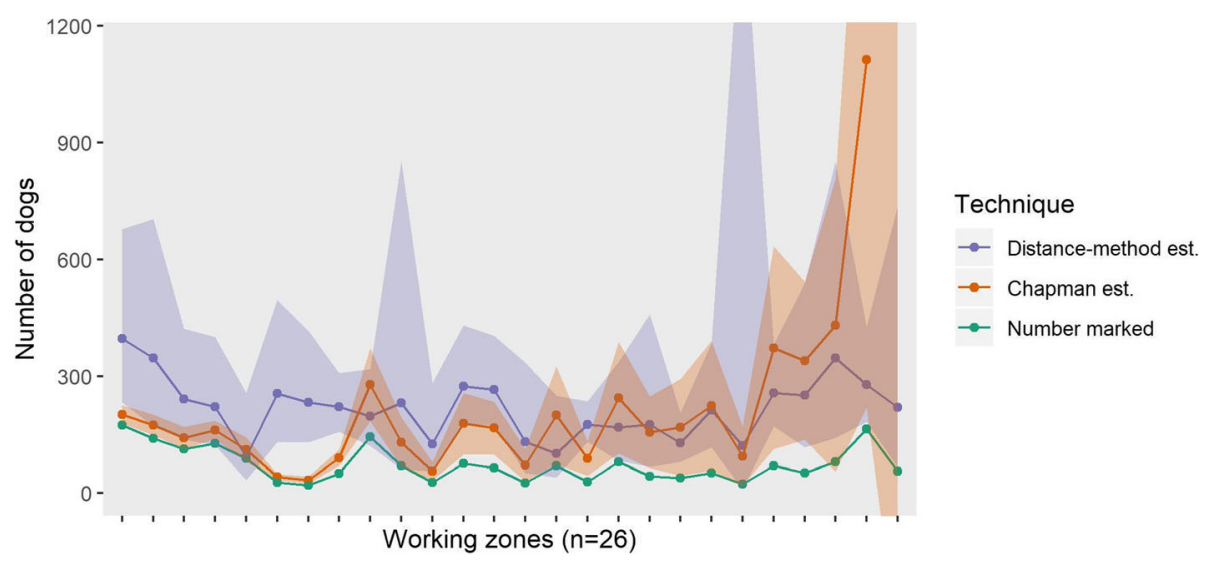

Fig. 4 Number of dogs estimated per working zone ordered in decreasing marking proportion. Confidence intervals (shaded area) for the distance sampling method estimate (circle) and Chapman mark-resight estimate (triangle). The number of dogs initially marked (vaccinated) per area (diamond) is also given

(95\%CI 245-314) for the subset of roads, and 8 dogs (SD 2.7) were seen on average per $\mathrm{km}$ of road covered.

When comparing the surveys that covered all roads versus those that only covered the subset of roads, the Chapman estimate mean difference was 0.45 (95\%CI $-56.96,57.85)$ and the CCC was 0.41 indicating moderate agreement. There was marked variation at higher estimates as seen in Fig. 5b. For the distance sampling method estimates, the mean difference was 32 dogs fewer $(95 \% \mathrm{CI}-61.83,-2.91)$ for all roads compared to the subset surveys, and the CCC was 0.37 (Table 3).

\section{Discussion}

Across the total study area, the distance sampling method gave comparable abundance estimates to the Chapman method. This supports the possibility that distance sampling may prove to be a cost and resource efficient method for estimating free-roaming dog populations but requires more studies over larger areas. There was variation at the working zone level but many areas still had overlapping confidence intervals between methods (Fig. 4). While some of this variation can be explained by differences in surveyors, these differences may also be due to expected natural variability in dog sightings.

Belo et al., (2017) observed that individual census counts do not adequately estimate abundance, as there are differences in dog detection [27]. The number of dogs counted in each working zone was small on average, and the proportion of marked dogs and corresponding Chapman estimate, would be heavily influenced by this, which is a major limitation of this study. It is also
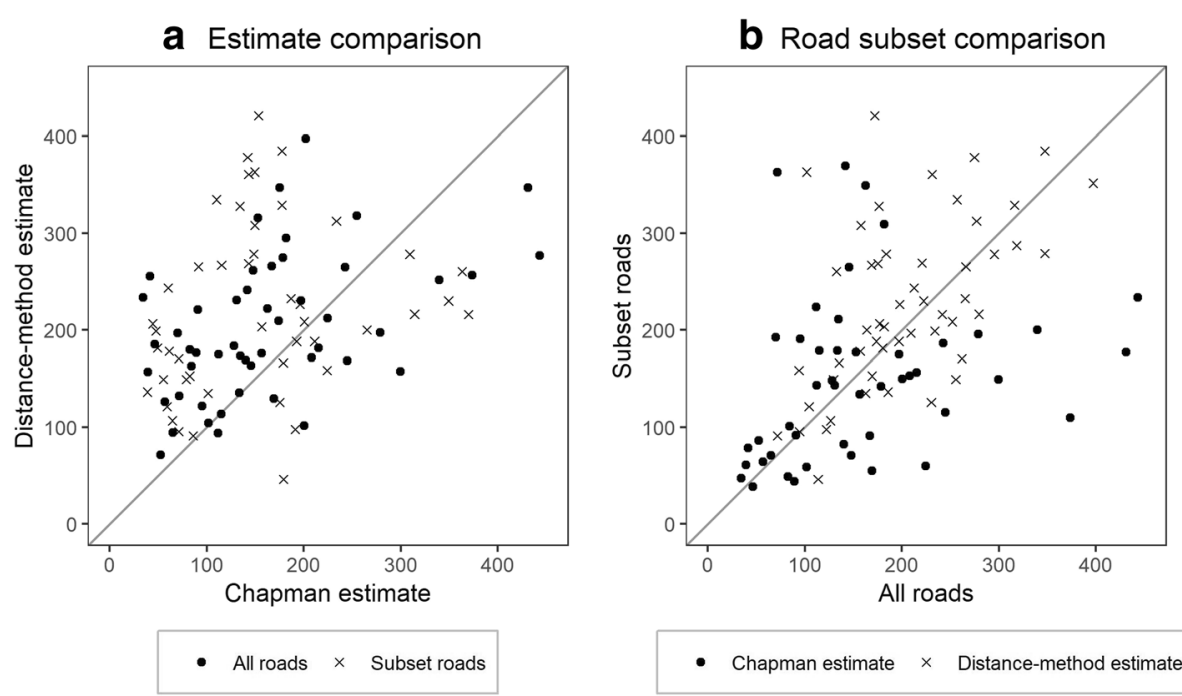

- Chapman estimate $\times$ Distance-method estimate

Fig. 5 Plot comparing, a) Chapman vs distance sampling method estimates, and b) estimates from all roads vs subset of roads 
Table 2 Estimates from the mixed effects model, showing fixed and random effects, as well as the intraclass correlation coefficient (ICC) for the random effects. The log transformed abundance estimate was modelled as the outcome. Coefficients are exponentiated and $p$-values given

\begin{tabular}{llll}
\hline Fixed effects & EXP( $\beta)$ & $95 \% \mathrm{Cl}$ & P-value \\
Intercept & 157.83 & $(125.47-198.41)$ & $<0.001$ \\
$\begin{array}{l}\text { Distance-method } \\
\text { vs Chapman }\end{array}$ & 1.35 & $(1.20-1.53)$ & $<0.001$ \\
Surveyor B vs A & 1.21 & $(1.07-1.37)$ & $<0.01$ \\
Survey 2 vs survey1 & 0.82 & $(0.72-0.92)$ & $<0.01$ \\
Subset vs all roads & 1.05 & $(0.92-1.19)$ & 0.48 \\
Afternoon vs morning & 0.80 & $(0.57-1.11)$ & 0.19 \\
Random effects & Variance & Std. Dev. & ICC \\
Working zones & 0.14 & 0.38 & 0.41 \\
Residual & 0.20 & 0.45 & \\
\hline
\end{tabular}

not known for how long the initial disturbance of the capture operation will influence the resident dog population to disperse out of the area. At the working zone level, there was evidence that the distance sampling method estimates were higher compared to the Chapman estimate when adjusting for surveyors, surveys, time and type of survey. This was more noticeable when looking only at the subset of roads. There was no evidence from the statistical model of a difference between the estimates from all roads versus the subset of roads, although the subset of roads produced more uncertainty. The actual number of dogs counted per survey was low compared to the estimates, and direct counts for the purpose of a census would not be recommended, except as an indicator count.

This study estimated an average dog density of 242 dogs per $\mathrm{km}^{2}(95 \% \mathrm{CI}$ 213-275) which was higher than Mumbai, 57 dogs per $\mathrm{km}^{2}$ [28], and Katwa, 178 dogs per $\mathrm{km}^{2}$ [29], and within the range of densities reported in other countries [14]. The higher densities reported here compared to other Indian studies may be due to this study limiting the study area to within $100 \mathrm{~m}$ of the road network.
Considering the density of dogs per $\mathrm{km}$ of road, this study reported an average of 6 dogs (SD 1.9) per $\mathrm{km}$ of road covered, which was lower than a study in Nepal, but higher than Latin American estimates [30]. Again, these estimates are highly variable dependant on location. A higher density of dogs was seen in town neighbourhoods compared to rural areas in a Bhutan study [22], and this variation between studies may be accounted for by housing density, geographic features, land use, or survey method [14].

Assumptions for the mark-resight method used here, include a closed population and no mark loss. Resight surveys were conducted within $48 \mathrm{~h}$ of the vaccination and marking. This short time interval should allow for minimal population changes for the closed population assumption to be valid. While dogs generally stay near their homes, some dogs can have large home ranges [31] and the borders of our working zones were arbitrary. Movement into and out of the study areas is therefore possible but was difficult to measure. This study did not account for mark loss due to the short time interval of less than 5 days, however it was possible for misclassification to occur. Conan et al., (2015) saw difficulties with darkly coloured dogs as well as misclassification of colours [32]. Additionally, dogs may have been doublecounted within the same survey if they moved, as individual identification of animals was not attempted.

A benefit of the distance sampling survey technique, is that no catching and marking is required, meaning that it is a much less resource intensive and welfare friendly approach. Permanent marking, such as ear notches have been used for dog population estimates [33] but they require accurate measurement of the number of notched dogs released, which is not always possible in field situations. Beck, (1973) popularised a method requiring photographic comparison of dogs to identify individuals, rather than physical marking [15]. While this method is useful as it does not require resource intensive catching of dogs, in large scale operations it may be impractical to manually match photographs. Pattern recognition software is available for species such as wild dog and

Table 3 Method comparison showing the mean difference between methods, Lin's Concordance Correlation Coefficient (CCC), and Pearson's correlation coefficient. The abundance estimates between the Chapman and distance sampling methods is compared; as well as the abundance estimates, marked proportion seen, and number of dogs counted per survey for the comparison of all roads to the subset

\begin{tabular}{lllllll}
\hline Comparison & \multicolumn{2}{l}{ Mean difference } & \multicolumn{2}{l}{ Lin's CCC } & & Pearson's R \\
\hline $\begin{array}{l}\text { Chapman vs distance-method } \\
\text { All roads vs subset }\end{array}$ & -20.76 & $(-58.53,17.01)$ & 0.17 & $(0.01,0.31)$ & 0.21 & $(0.01,0.38)$ \\
$\quad$ & & & & & \\
$\quad$ Chapman estimate & 0.45 & $(-56.96,57.85)$ & 0.41 & $(0.17,0.61)$ & 0.43 & $(0.17,0.63)$ \\
$\quad$ Distance-method estimate & -32.37 & $(-61.83,-2.91)$ & 0.37 & $(0.15,0.55)$ & 0.43 & $(0.18,0.63)$ \\
$\quad$ Marked proportion & 0.03 & $(-0.06,0.13)$ & -0.12 & $(-0.37,0.16)$ & -0.12 & $(-0.38,0.16)$ \\
$\quad$ Number of dogs & 8.98 & $(6.84,11.12)$ & 0.33 & $(0.18,0.46)$ & 0.61 & $(0.40,0.76)$ \\
\hline
\end{tabular}


giraffe with characteristic coat patterns [34]. This would need to be validated for domestic dogs which have fewer discriminatory patterns to be used in large scale photographic surveys.

The distance sampling method assumptions include random placement of the survey lines which are, by definition, not valid when travelling along roads for the survey. Roads and human activity are likely to be associated with more dogs, resulting in an overestimation. We purposively subset the study area to within $100 \mathrm{~m}$ of known roads, to minimise the overestimation in areas unlikely to include dogs. The areas outside of the $100 \mathrm{~m}$ buffer were visually confirmed on satellite images to be areas with few to no buildings. Despite this, our estimates for the distance sampling methods may have reflected this overestimation and were, on average per working area, higher than the Chapman mark-resight methods. When considering estimates for the entire study area, however, there was overlap in the confidence intervals between methods. Another limitation of the distance sampling method, is that we did not take group size into account in the final analysis due to data reliability issues. Dogs were often seen roaming in groups and this may have affected the estimates.

There is added complexity to these surveys as many owned dogs may have variable roaming patterns. For example, dogs may be restricted during the time of marking (vaccination) and therefore not taken into account as a marked free-roaming dog, and then later allowed to roam freely during the resight surveys and considered free-roaming. It is unknown in what direction this may affect estimates, and household surveys may help address this. As this study only looked at free-roaming dogs, a household survey would be needed in any case for a complete picture of the dog population.

This study did not explicitly look at the interpretation of the marked proportion of dogs as vaccination coverage. However, the data would suggest that the vaccination coverage in this population is lower than the targeted figure of $70 \%$. As part of the campaign, additional follow-up vaccination drives would have taken place in areas with lower coverage, which are not reflected in this study. Secondly, this study represents a subset of the $\operatorname{dog}$ population in the area, i.e. freeroaming dogs only, and does not take into account confined dogs. The vaccination coverage in unowned, freeroaming dogs may be lower than the general population due to difficulties in catching dogs that are not used to being handled but has not been routinely studied. This subset of the population may need alternative vaccination efforts, such as oral bait vaccine, to ensure adequate coverage.

Initial dog population estimates are important for planning the resources, time and funds needed for pilot dog vaccination or population management initiatives. However, this should be off-set against diverted limited resources that could be invested in the intervention. In areas where there is limited or no data about the dog population, the use of efficient survey methods enables initial population estimates to be calculated without detracting resources from the intervention. Data from initial pilot work can then be used to refine population estimates [12].

\section{Conclusion}

In summary, the distance sampling method was adequately comparable to Chapman mark-resight in this study for the purpose of overall population estimates of free-roaming dogs across large areas containing multiple working zones, despite variation in abundance estimates within individual working zones. Although the distance sampling method requires more computational expertise, it could be valuable for resource limited control programmes if shown to give similar comparability in other regions over large areas. Systems which automate the computational components of estimating the wider population may make such methods more accessible in resource limited settings. Additionally, using only a representative number of roads to conduct the resight surveys may further save resources with an acceptable level of uncertainty in the population abundance estimation.

\section{Additional files}

Additional file 1: Tables S1. Final detection function models used to estimate dog density and abundance using the Distance package in $\mathrm{R}$. (DOCX $12 \mathrm{~kb}$ )

Additional file 2: Figure S1. Bland-Altman plots are given for the difference between Chapman and distance-method estimates, as well as the difference between methods for all roads and the subset of roads. (DOCX $177 \mathrm{~kb})$

\section{Abbreviations}

AIC: Akaike information criterion; CCC: Concordance correlation coefficient; GPS: Global Positioning System; WS: Worldwide Veterinary Services

\section{Acknowledgements}

The authors wish to acknowledge the surveyor team at Mission Rabies assisting in the data collection for this study.

\section{Authors' contributions}

NM and RM lead all aspects of this study. AG, JC, MB, LG assisted with the design and planning. $\mathrm{IH}$ and SM assisted with design and analysis. The field work was managed by JC and NM. The final manuscript was contributed to and reviewed by all authors. All authors read and approved the final manuscript.

\section{Funding}

This study was performed in collaboration with Mission Rabies and the Worldwide Veterinary Service, sponsored by Dogs Trust and the Marchig Animal Welfare Trust. NM is supported by the Marchig Animal Welfare Trust and Dogs Trust. $M B$ receives support from the BBSRC as part of the RI core strategic funding (BB/J004235/1). The funders played no role in the design of 
the study and collection, analysis, and interpretation of data and in the writing of the manuscript.

\section{Availability of data and materials}

The datasets used during the current study are available from the corresponding author on reasonable request and with the permission of Mission Rabies. Map data is adapted from GADM for Fig. 1. https://gadm.org. The cartography in Fig. 2 has been adapted from OpenStreetMap under the Creative Commons Attribution-ShareAlike 2.0 license (CC BY-SA). https:// www.openstreetmap.org/copyright

\section{Ethics approval and consent to participate}

Ethics approval was attained from the University of Edinburgh, Veterinary Ethics Research Committee (VERC 7.18).

\section{Consent for publication}

Permission to publish data from the Goa rabies vaccination campaign was given by the Government of Goa Department for Animal Husbandry.

\section{Competing interests}

The authors declare that they have no competing interests.

\section{Author details}

${ }^{1}$ The Royal (Dick) School of Veterinary Studies (R(D)SVS) and the Roslin Institute, Hospital for Small Animals, Easter Bush Veterinary Centre, Midlothian EH25 9RG, Scotland. 'ªission Rabies, 4 Castle Street, Cranborne, Dorset BH21 5PZ, UK. ${ }^{3}$ The Epidemiology, Economics and Risk Assessment (EERA) Group, The Roslin Institute and the Royal (Dick) School of Veterinary Studies (R(D)SVS), Easter Bush, Midlothian EH25 9RG, Scotland.

\section{Received: 8 January 2019 Accepted: 29 May 2019}

Published online: 07 June 2019

\section{References}

1. Sudarshan MK, Madhusudana SN, Mahendra BJ, Rao NSN, Ashwath Narayana DH, Abdul Rahman S, et al. Assessing the burden of human rabies in India: results of a national multi-center epidemiological survey. Int J Infect Dis. 2007;11(1):29-35.

2. Cleaveland S, Kaare M, Tiringa P, Mlengeya T, Barrat J. A dog rabies vaccination campaign in rural Africa: impact on the incidence of dog rabies and human dog-bite injuries. Vaccine. 2003;21(17-18):1974-82.

3. WHO. WHO Expert Consultation on Rabies, 5-8 October 2004 [Internet]. 2005. Available from: TRS 931, WHO 2005.

4. Totton SC, Wandeler Al, Gartley CJ, Kachhawaha S, Suman M, Ribble CS, et al. Assessing reproductive patterns and disorders in free-ranging dogs in Jodhpur, India to optimize a population control program. Theriogenology [Internet]. 2010;74(7):1115-1120. Available from: https://doi.org/10.1016/j. theriogenology.2010.05.008

5. Coleman PG, Dye C. Immunization coverage required to prevent outbreaks of dog rabies. Vaccine. 1996;14(3):185-6.

6. Davlin SL, VonVille HM. Canine rabies vaccination and domestic dog population characteristics in the developing world: a systematic review. Vaccine. 2012;30(24):3492-502.

7. Belsare A V., Gompper ME. Assessing demographic and epidemiologic parameters of rural dog populations in India during mass vaccination campaigns. Prev Vet Med [Internet]. 2013;111(1-2):139-146. Available from: https://doi.org/10.1016/j.prevetmed.2013.04.003

8. WSPA. Surveying roaming dog populations: guidelines on methodology. Companion \& Working Animals Unit. 2012.

9. Lembo T. The blueprint for rabies prevention and control: a novel operational toolkit for rabies elimination. PLoS Negl Trop Dis. 2012;6(2):1-4.

10. Hiby E, Atema KN, Brimley R, Hammond-Seaman A, Jones M, Rowan A, et al. Scoping review of indicators and methods of measurement used to evaluate the impact of dog population management interventions; 2017. p. 1-20.

11. Reece JF, Chawla SK. Control of rabies in Jaipur, India, by the sterilisation and vaccination of neighbourhood dogs. Vet Rec. 2006;159(12):379-83.

12. Gibson AD, Handel IG, Shervell K, Roux T, Mayer D, Muyila S, et al. The vaccination of 35,000 dogs in 20 working days using combined static point and door-to-door methods in Blantyre, Malawi. PLoS Negl Trop Dis [Internet]. 2016;10(7):e0004824. Available from: https://doi.org/10.1371/ journal.pntd.0004824
13. Dalla Villa P, Kahn S, Stuardo L, lannetti L, Di Nardo A, Serpell JA. Freeroaming dog control among OIE-member countries. Prev Vet Med [Internet]. 2010;97(1):58-63. Available from: https://doi.org/10.1016/j. prevetmed.2010.07.001

14. Belo VS, Werneck GL, Da Silva ES, Barbosa DS, Struchiner CJ. Population estimation methods for free-ranging dogs: a systematic review. PLoS One. 2015;10(12):1-15.

15. Beck AM. The ecology of stray dogs: a study of free-ranging animals [internet]. Purdue: University Press; 1973. Available from: http://docs.lib. purdue.edu/press_ebooks/4

16. Fei SY, Chiang JT, Fei CY, Chou CH, Tung MC. Estimating stray dog populations with the regression method versus Beck's method: a comparison. Environ Ecol Stat. 2012;19(4):485-98.

17. Tiwari HK, Vanak AT, O'Dea M, Gogoi-Tiwari J, Robertson ID. A Comparative Study of Enumeration Techniques for Free-Roaming Dogs in Rural Baramati, District Pune, India Front Vet Sci [Internet]. 2018;5:112. Available from: https://www.frontiersin.org/article/10.3389/fvets.2018. 00104/full

18. Childs JE, Robinson LE, Sadek R, Madden A, Miranda ME, Miranda NL. Density estimates of rural dog populations and an assessment of marking methods during a rabies vaccination campaign in the Philippines. Prev Vet Med [Internet]. 1998;33(1-4):207-218. Available from: http://www. sciencedirect.com/science/article/pii/S0167587797000391

19. Gibson AD, Ohal P, Shervell K, Handel IG, Bronsvoort BM, Mellanby RJ, et al. Vaccinate-assess-move method of mass canine rabies vaccination utilising mobile technology data collection in Ranchi, India. BMC Infect Dis [Internet]. 2015;15(1):589 Available from: http://www.biomedcentral.com/1471-2334/ $15 / 589$

20. Gibson AD, Mazeri S, Lohr F, Mayer D, Burdon JL, Wallace RM, et al. One million dog vaccinations recorded on mHealth innovation used to direct teams in numerous rabies control campaigns. 2018;1-19.

21. OpenStreetMap Contributors. Retrieved from https://planet.osm.org [Internet]. 2018. Available from: https://www.openstreetmap.org.

22. Tenzin T, Ahmed R, Debnath NC, Ahmed G, Yamage M. Free-roaming dog population estimation and status of the dog population management and rabies control program in Dhaka City, Bangladesh. PLoS Negl Trop Dis [Internet]. 2015;9(5):1-14. Available from. https://doi.org/10.1371/journal. pntd.0003784.

23. Miller DL, Rexstad E, Thomas L, Marshall L, Laake J. Distance sampling in R. bioRxiv [Internet]. 2016;063891. Available from: https://doi.org/10.1101/ 063891

24. QGIS Development Team. QGIS Geographic Information System [Internet]. Open Source Geospatial Foundation Project; 2018. Available from: http:// qgis.osgeo.org

25. Bland JM, Altman DG. Statistical methods for assessing agreement between two methods of clinical measurement. Lancet [Internet]. 1986;327:307-310. Available from: http://www.sciencedirect.com/science/article/pii/ S0140673686908378

26. Watson PF, Petrie A. Method agreement analysis: a review of correct methodology. Theriogenology [Internet]. 2010;73(9):1167-1179. Available from: https://doi.org/10.1016/j.theriogenology.2010.01.003

27. Belo VS, Struchiner CJ, Werneck GL, Teixeira Neto RG, Tonelli GB, De Carvalho CG, et al. Abundance, survival, recruitment and effectiveness of sterilization of free-roaming dogs: a capture and recapture study in Brazil. PLoS One. 2017;12(11):1-19.

28. Punjabi GA, Athreya $V$, Linnell JDC. Using natural marks to estimate freeranging dog canis familiaris abundance in a MARK-RESIGHT framework in suburban Mumbai, India. Trop Conserv Sci. 2012;5(4):510-20.

29. Pal SK. Population ecology of free-ranging urban dogs in West Bengal, India. Acta Theriol (Warsz). 2001:46(1):69-78.

30. Hiby E, Hiby L. Direct observation of dog density and composition during street counts as a resource efficient method of measuring variation in roaming dog populations over time and between locations. Animals. 2017;7(8).

31. Dürr S, Dhand NK, Bombara C, Molloy S, Ward MP. What influences the home range size of free-roaming domestic dogs? Epidemiol Infect. 2017; 145(7):1339-50.

32. Conan A, Kent A, Koman K, Konink S, Knobel D. Evaluation of methods for short-term marking of domestic dogs for rabies control. Prev Vet Med [Internet]. 2015;121(1-2):179-182. Available from: https://doi.org/10.1016/j. prevetmed.2015.05.008 
33. Hiby LR, Reece JF, Wright $R$, Jaisinghani $R$, Singh B, Hiby EF. A mark-resight survey method to estimate the roaming dog population in three cities in Rajasthan, India. BMC Vet Res [Internet]. 2011;7(1):46. Available from: http:// www.biomedcentral.com/1746-6148/7/46

34. Bolger DT, Morrison TA, Vance B, Lee D, Farid H. A computer-assisted system for photographic mark-recapture analysis. Methods Ecol Evol. 2012; 3(5):813-22

\section{Publisher's Note}

Springer Nature remains neutral with regard to jurisdictional claims in published maps and institutional affiliations.

Ready to submit your research? Choose BMC and benefit from:

- fast, convenient online submission

- thorough peer review by experienced researchers in your field

- rapid publication on acceptance

- support for research data, including large and complex data types

- gold Open Access which fosters wider collaboration and increased citations

- maximum visibility for your research: over $100 \mathrm{M}$ website views per year

At $\mathrm{BMC}$, research is always in progress.

Learn more biomedcentral.com/submissions 